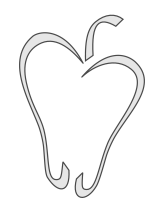

ISSN $1852-4249$

\title{
Development of two-stage multi-anvil apparatus for low-temperature measurements
}

\begin{abstract}
K. Ishigaki, ${ }^{1 *}$ J. Gouchi,${ }^{1}$ S. Nagasaki, ${ }^{1}$ J. G. Cheng, ${ }^{2}$ Y. Uwatoko ${ }^{1 \dagger}$
The two-stage 6-8 multi-anvil (MA8) apparatus is an important large-volume, highpressure technique that has been widely used in the high pressure mineralogy and material synthesis, mainly at room temperature or above. Recently, we have successfully developed a two-stage MA8 apparatus for low-temperature physical property measurements. The first-stage anvils at top and bottom sides are fabricated as a single piece in order to reduce the total size of the cylindrical module, which is put in a top-loading high pressure cryostat and compressed by a 1000 ton hydraulic press. A castable, split octahedral gasket with integrated fin was specifically designed in order to introduce the electrical leads from the inside sample container filled with a liquid pressure transmitting medium. By using tungsten carbide (WC) second-stage cubes with a truncated edge length of $3 \mathrm{~mm}$ and an octahedral gasket with an edge length of $6 \mathrm{~mm}$, we have successfully generated pressure over $20 \mathrm{GPa}$ at room temperature. Since the high pressure limit can be pushed to nearly $100 \mathrm{GPa}$ by using the sintered diamond second-stage cubes, our MA8 apparatus has a great potential to expand the current pressure capacity for precise low-temperature measurements with a large sample volume.
\end{abstract}

\section{Introduction}

Pressure is a fundamental parameter like temperature that governs the states of matter. The application of high pressure can induce structural or electronic phase transitions or precisely tune the structural and physical properties. In condensed matter physics, the combination of high-pressure and low-temperature environments provides a very fertile ground for exploring novel quantum states of matter and exotic phenomena. For example, pres-

\footnotetext{
*E-mail: ishigaki@issp.u-tokyo.ac.jp

†E-mail: uwatoko@issp.u-tokyo.ac.jp

1 Institute for Solid State Physics, University of Tokyo, 5-1-5 Kashiwanoha, Chiba 277-8581, Japan.

2 Institute of Physics, Chinese Academy of Sciences, Beijing 100190, People's Republic of China.
}

sure can induce a magnetic quantum critical point, near which the Landau Fermi-liquid behavior usually breaks down and unconventional superconductivity frequently takes place due to the presence of strong quantum fluctuations. Therefore, it is important to develop a high pressure apparatus for low-temperature measurements.

Despite the sophisticated low-temperature technologies existent, the high-pressure devices used in low-temperature conditions remain to be further developed due to the space constrain and other specific requirements, such as pressure homogeneity, sample volume, etc. Currently, piston-cylinder cell (PCC) [1,2] and diamond anvil cell (DAC) [3,4] are two widely used commercial high-pressure devices for in-situ physical property measurements at low temperatures. PCC offers a large sample space and relatively good hydrostaticity by employing a liquid 
pressure transmitting medium (PTM) [5], but the maximum pressure is usually limited to $4 \mathrm{GPa}$ [1], which is insufficient for many studies in condensed matter. Although the DAC [3] can achieve ultrahigh pressures and allow easy access for the electromagnetic radiations, the tiny sample space makes it difficult for in-situ physical property measurements requiring electrical contacts, and the solid PTM usually employed renders severe non-hydrostatic pressure conditions.

Besides the PTM, the level of pressure hydrostaticity/homogeneity also depends on the compression geometry. In comparison with DAC, multianvil-type (MA) apparatus can maintain better pressure homogeneity even if the PTM becomes solidified at low temperature and/or high pressure [6]. In addition, the MA apparatus can reach pressure above $10 \mathrm{GPa}$, much higher than PCC. The single-stage cubic anvil cell (CAC) device developed in the Institute for Solid State Physics, the University of Tokyo (ISSP, UT) [7] is one typical MA apparatus that can generate hydrostatic pressures up to $15 \mathrm{GPa}$. The design of miniature "palm"-type CAC also enabled integration with ${ }^{3} \mathrm{He}$ or dilution refrigerator so as to reach temperatures as low as $10 \mathrm{mK}[8,9]$. These developments of cubic-type apparatus were essential for us to discover novel quantum phenomena [10] and new superconducting materials [11] recent years.

To pursue more exotic phenomena in an extended pressure range, there is always a demand for the development of devices reaching even higher pressures. In this regard, the two-stage 6-8 multianvil (MA8) apparatus originally developed in 1970s by Kawai and Endo becomes an excellent option [12]. In this case, the first stage of six anvils surrounds a cubic cavity, in which it is placed the second stage, consisting of eight cubes with truncated corners forming an octahedron. After 40 years of developments, the MA8 apparatus has gain great success and has been widely used in high-pressure mineralogy and synthesis of materials. Depending on the strength of the secondstage anvils, the maximum pressure of MA8 are used for high-pressure studies at or above room temperature. In this paper, we report the development of a two-stage MA8 apparatus for precise low-temperature physical property measurements in ISSP, UT.
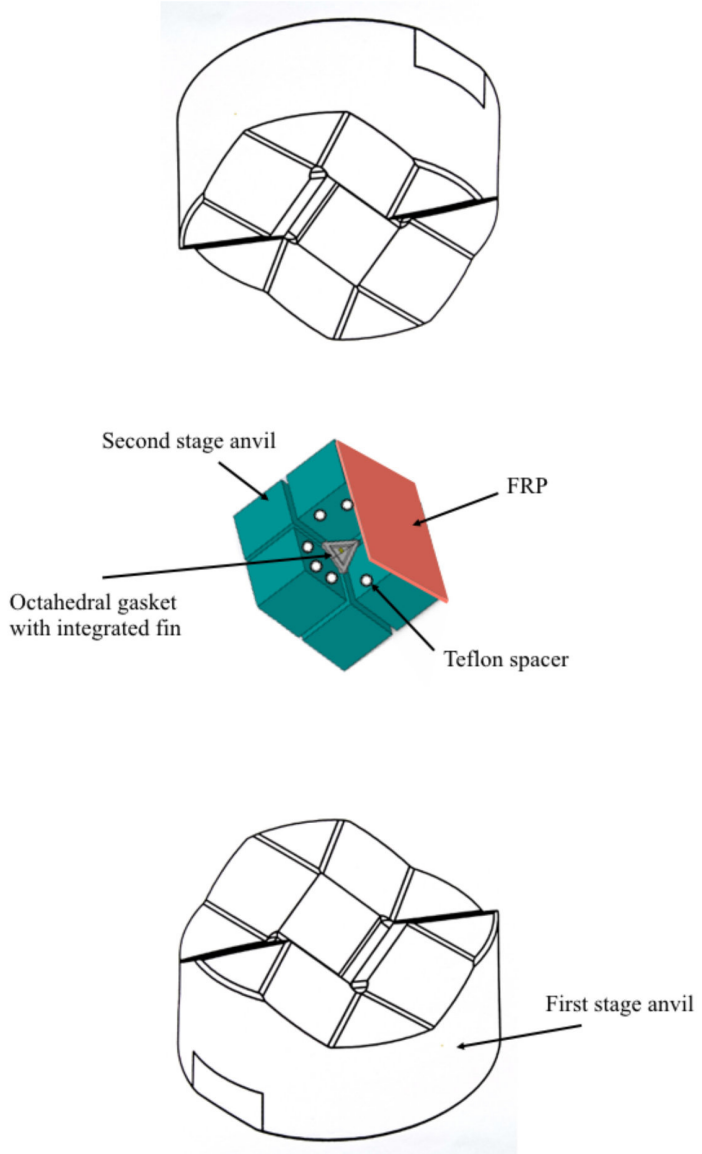

Figure 1: Schematic illustration of the first-stage and second-stage anvils.

\section{Experimental setup and results}

\section{i. Two-stage MA8 device}

For the commonly used two-stage MA8 apparatus, the first-stage six anvils (three on the top and three on the bottom) made of hardened steel are usually built into a thick-wall steel ring (Kawai type) or contained in a removable cylindrical module (Walker type) [13]. Such designs are not suitable for low-temperature applications because the whole MA8 device has to be inserted into a cryostat. To reduce the total size of the MA8 device, we designed the first-stage three anvils on top and bottom sides as a whole piece, as shown in Fig. 1. We have also used a nonmagnetic $\mathrm{NiCrAl}$ alloy to fab- 


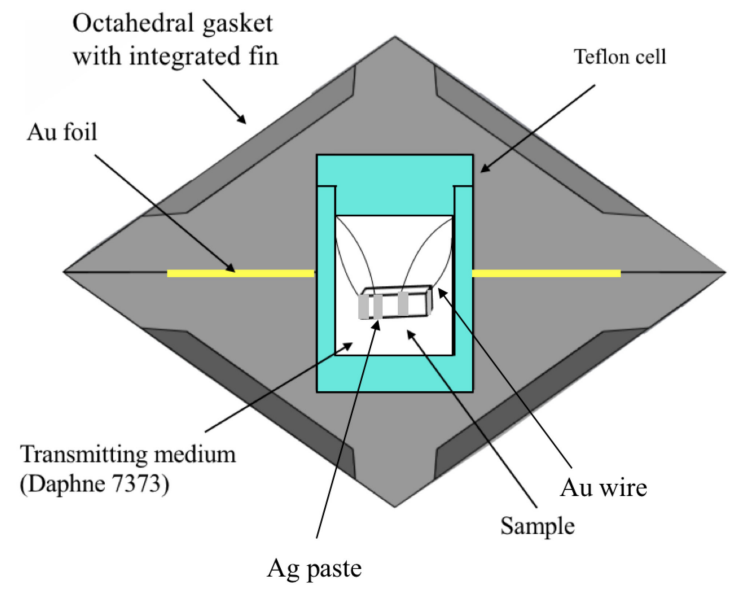

Figure 2: Cross-sectional view of the internal configuration of the gasket with teflon cell.

ricate the pair of cylindrical first-stage anvils in order to apply magnetic fields. The first-stage anvils have an outer diameter of $154 \mathrm{~mm}$ and form a cubic cavity with edge length of $32.3 \mathrm{~mm}$. The secondstage anvils, consisting of eight cubes with truncated corners, are similar to the commonly used MA8 apparatus. Here, we employed nonmagnetic WC (TMS05/MF10 grade from Fujilloy) with an edge length of $18 \mathrm{~mm}$ and truncated corner of 3 $\mathrm{mm}$. As a common practice, these $\mathrm{WC}$ cubes are held together with six pieces of Fiber-Reinforced Plastics (FRP) pads, which are $0.5 \mathrm{~mm}$ in thickness and $36 \times 36 \mathrm{~mm}$ in area. These FRP pads also serve as an insulation to the first-stage anvils. The inside surfaces of the second-stage cubes are pasted with three $1.0 \mathrm{~mm}$ cubic Teflon spacers to prevent electrical contact with adjacent anvils.

\section{ii. Gasket design and sample assembly}

The adoption of a liquid PTM is essential to maintain a relatively good pressure homogeneity. However, the conventional design of octahedral gasket and sample assembly used for the MA8 apparatus also need to be modified in order to accommodate a sample container filled with liquid PTM. For this purpose, we adopt the castable, split octahedral gasket with integrated fin, which are made from Ceramacast 584-P and Ceramacast 584-L (100:28

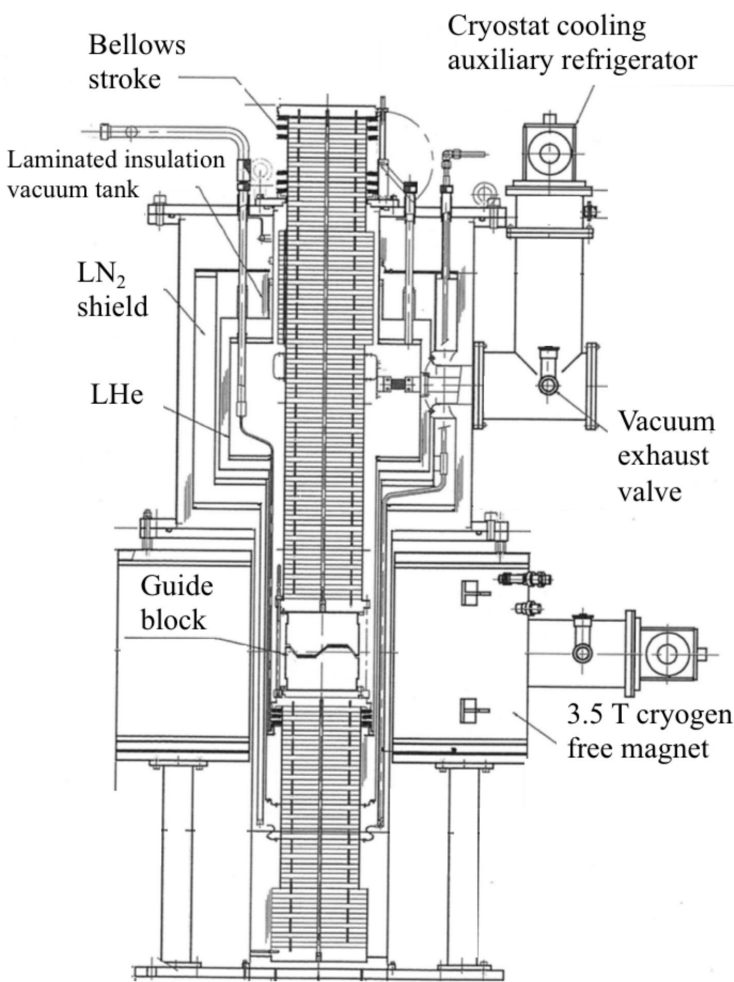

Figure 3: Cross-sectional view of the top-loading cryostat.

weight-in-weight) potting compound from Aremco Products, Inc. The half-octahedral gaskets with integrated fins are made in-house in our laboratory according to the procedures described in Ref. [14]. The edge length of the octahedron is $6 \mathrm{~mm}$ and the thickness of the gasket fin is $1 \mathrm{~mm}$. Figure 2 depicts the internal configuration of the gasket with the sample hanging inside the Teflon capsule (I.D. $1.5 \mathrm{~mm}$, O.D. $2.0 \mathrm{~mm}$ and length $2.5 \mathrm{~mm}$ ), which is the same setup used in the cubic anvil cell [7]. The Teflon cell can be filled with a liquid PTM such as Daphne 7373 or Glycerol, and the electrical leads are introduced via gold foil to the surfaces of octahedral gasket, which in turn contact with the WC cubes.

\section{iii. Top loading high-pressure cryostat}

Figure 3 shows a schematic cross-sectional view of the top-loading high-pressure cryostat, in which 
PAPERs IN Physics, vol. 11, ART. 110006 (2019) / K. Ishigaki et al.

Table 1: Phase transitions as pressure calibrants [15].

\begin{tabular}{lc}
\hline Sample & Pressure $(\mathrm{GPa})$ \\
\hline \hline $\mathrm{Bi}$ & $2.55,2.7,7.7$ \\
$\mathrm{Sn}$ & 9.4 \\
$\mathrm{~Pb}$ & 13.4 \\
$\mathrm{ZnS}$ & 15.6 \\
$\mathrm{GaAs}$ & 18.3 \\
\hline
\end{tabular}

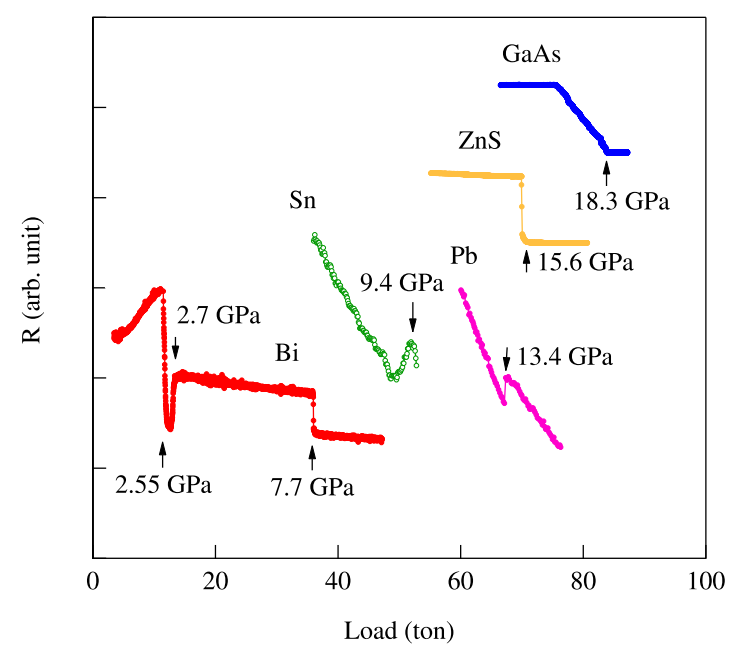

Figure 4: Electrical Resistance of $\mathrm{Bi}, \mathrm{Sn}, \mathrm{Pb}, \mathrm{ZnS}$ and GaAs as a function of loading force.

the MA8 device is placed in between the upper and lower pushing columns. Details about the design of the high-pressure cryostat can be found in an earlier publication about the cubic anvil cell apparatus [7]. The low-temperature condition (down to $2 \mathrm{~K}$ ) is realized by filling the cryostat with liquid nitrogen and then helium with proper pumping. Precise temperature control between 2 and $300 \mathrm{~K}$ was achieved by attaching a resistance heater onto the MA8 device. The pressure is generated by using a 1000-ton hydraulic press, which can maintain a constant loading force over the MA8 device over the whole temperature range. In addition, a 3.5 Tesla helium-free superconducting magnet with a large bore size is also installed and the center of the magnetic field is aligned with the sample in the MA8 device.

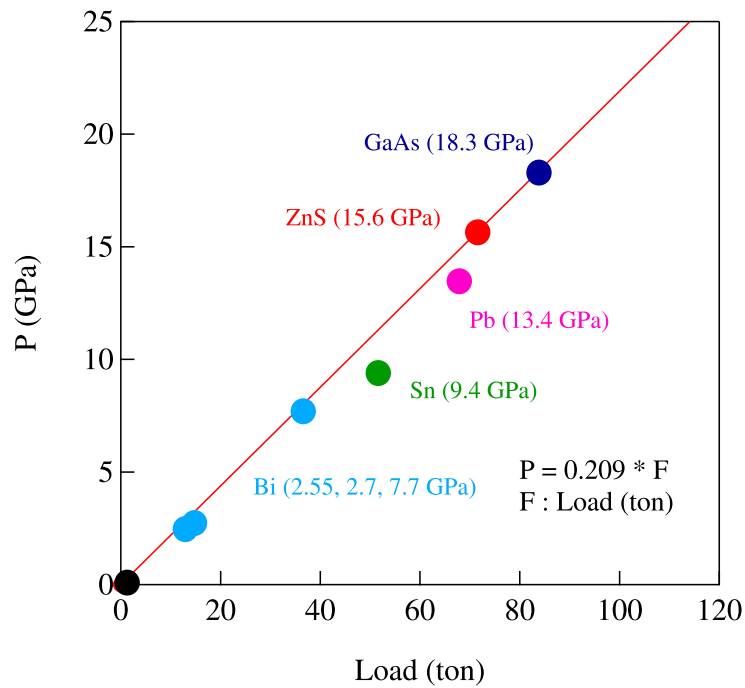

Figure 5: Pressure calibration line for a two-stage multi-anvil high pressure cell.

\section{iv. Pressure calibration}

We have performed fixed point pressure calibration at room temperature by detecting the characteristic phase transitions of $\mathrm{Bi}, \mathrm{Sn}, \mathrm{Pb}, \mathrm{ZnS}$ and $\mathrm{GaAs}$ in electrical resistance. A standard fourprobe method was used to measure the resistance of each sample. Table 1 summarizes the transition pressure of these materials from previous studies [15].

Figure 4 shows the electrical resistance of $\mathrm{Bi}, \mathrm{Sn}$, $\mathrm{Pb}, \mathrm{ZnS}$ and GaAs as a function of loading force at room temperature. As can be seen, the characteristic phase transitions of $\mathrm{Bi}$ at 2.55, 2.7 and 7.7 GPa were clearly observed at loading force of $12.2,13.7$, and 36.2 tons, respectively. We defined the phase transitions which are the offset. Similarly, the resistance anomalies of $\mathrm{Sn}$ and $\mathrm{Pb}$ at 9.4 and $13.4 \mathrm{GPa}$ were also observed at 51.7 and 67.9 tons, respectively. In addition, the metallization of $\mathrm{ZnS}$ and GaAs at 15.6 and $18.3 \mathrm{GPa}$ were successfully observed at a loading force of 71.1 and 83.9 tons, respectively. Although the employed Daphne 7373 PTM becomes solid at about 2.3 GPa, these characteristic phase transitions remain very sharp, signaling an excellent pressure homogeneity up to at least $20 \mathrm{GPa}$ due to the multi-anvil geometry.

Based on these measurements, we have plotted 
PAPers in Physics, vol. 11, ART. 110006 (2019) / K. Ishigaki et al.

in Fig. 5 the pressure calibration curve for our twostage MA8 apparatus installed with WC cubes having a truncated corner of $3 \mathrm{~mm}$. As can be seen, all the calibration points fall nicely on a liner curve described by $P(\mathrm{GPa})=0.209 \times$ Force (ton). From the extrapolation, we can reach about $25 \mathrm{GPa}$ at a loading force of 120 tons, which is a much lower force than those reported in the literature employing $\mathrm{MgO}$ octahedron plus extra pyrophyllite gaskets. In the latter case, a large portion of loading force was dissipated on the relatively soft pyrophyllite gasket so that the calibration curve usually tends to saturate at higher loading forces. In contrast, the much improved pressure efficiency in our MA8 apparatus should be attributed to the octahedral gasket with integrated fin, which is much harder than pyrophyllite. As mentioned above, the maximum pressure at which MA8 can be pushed to is over $40 \mathrm{GPa}$ by using a tapered second-stage WC anvils [16], or to nearly $100 \mathrm{GPa}$ by employing much harder sintered diamond cubes [17]. It can be thus foreseen that the pressure capacity of our MA8 apparatus can be further improved.

\section{Conclusions}

We have successfully developed a two-stage 68 multi-anvil apparatus for accurate high-pressure and low-temperature measurements. By using tungsten carbide second-stage cubes with truncated corners of $3 \mathrm{~mm}$ and castable octahedral gasket with an edge length of $6 \mathrm{~mm}$, we can generate pressures over $20 \mathrm{GPa}$ at a relatively low loading force of 100 ton. An excellent pressure homogeneity/hydrostaticity up to $20 \mathrm{GPa}$ has been demonstrated in our MA8 apparatus, which is expected to reach even higher pressures by employing WC anvils with smaller truncation sizes or sintered diamond anvils.

Acknowledgements - This work was supported by Grant-Aid for Challenging Exploratory Research (No. 16K13830). JGC acknowledges the support from MOST, NSFC, and CAS through projects (Grant Nos. 2018YFA0305702, 1154377, 11874400, and QYZDB-SSW-SLH013).
[1] Y Uwatoko, S Todo, K Ueda, A Uchida, M Kosaka, N Mori, T Matsumoto, Material properties of $\mathrm{Ni}-\mathrm{Cr}-\mathrm{Al}$ alloy and design of a $4 \mathrm{GPa}$ class non-magnetic high-pressure cell, J. Phys.: Cond. Matter 14, 11291 (2002).

[2] N Fujiwara, T Matsumoto, K K Nakazawa, A Hisada, Y Uwatoko, Fabrication and efficiency evaluation of a hybrid NiCrAl pressure cell up to 4 GPa, Rev. Sci. Instr. 78, 073905 (2007).

[3] M Kano, H Mori, Y Uwatoko, S Tozer, Anisotropy of the upper critical field in ultrahigh-pressure-induced superconductor $(\text { TMTTF })_{2} P F_{6}$, Physica B: Cond. Matter 404, 3246 (2009).

[4] K Shimizu, H Ishikawa, D Takao, T Yagi, $\mathrm{K}$ Amaya, Superconductivity in compressed lithium at $20 \mathrm{~K}$, Nature 419, 597 (2002).

[5] K Murata, S Aoki, Development of high pressure medium achieving high quality hydrostatic pressure, Rev. High Pressure Sci. Technol. 26, 3 (2016).

[6] Y Nakamura, A Takimoto, M Matsui, Rheology and nonhydrostatic pressure evaluation of solidified oils including Daphne oils by observing microsphere deformation, J. Phys.: Conf. Series, 215, 012176 (2010).

[7] N Mori, H Takahashi, N Takeshita, Lowtemperature and high-pressure apparatus developed at ISSP, University of Tokyo, High Pressure Res. 24, 225 (2004).

[8] Y Uwatoko, K Matsubayashi, T Matsumoto, $\mathrm{N}$ Aso, M Nishi, $\mathrm{T}$ Fujiwara, M Hedo, $\mathrm{S}$ Tabata, K Takagi, M Tado, H Kagi, Development of palm cubic anvil apparatus for low temperature physics, Rev. High Pressure Sci. Technol. 18, 230 (2008).

[9] K Matsubayashi, A Hisada, T Kawae, Y Uwatoko, Recent progress in multi-extreme condition by miniature high-pressure, Rev. High Pressure Sci. Technol. 22, 206 (2012).

[10] K Matsubayashi, T Tanaka, A Sakai, S Nakatsuji, Y Kubo, Y Uwatoko, Pressureinduced heavy fermion superconductivity in the nonmagnetic quadrupolar system $\operatorname{PrTi}_{2} \mathrm{Al}_{2} \mathrm{O}$, Phys. Rev. Lett. 109, 187004 (2012). 
PApers in Physics, vol. 11, ART. 110006 (2019) / K. Ishigaki et al.

[11] J G Chen, K Matsubayashi, W Wu, J P Sun, M Nishi, F K Lin, J L Luo, Y Uwatoko, Pressure induced superconductivity on the border of magnetic order in MnP, Phys. Rev. Lett. 114, 117001 (2015).

[12] N Kawai, S Endo, The generation of ultrahigh hydrostatic pressures by a split sphere apparatus, Rev. Sci. Instr. 41, 1178 (1970).

[13] D Walker, M A Carpenter, C M Hitch, Some simplifications to multianvil devices for high pressure experiments, Am. Mineralog. 75, 1020 (1990).

[14] J G Cheng, High-pressure synthesis of the $4 d$ and $5 d$ transition-metal oxides with the perovskite and the perovskite-related structure and their physical properties, PhD. Thesis, University of Texas, Texas, Austin (2010).
[15] E Ito, Pressure calibration for multi-anvil apparatuses in high-pressure eatrth science, Rev. High Pressure Sci. Technol. 13, 265 (2003).

[16] T Ishii, L Shi, R Huang, N Tsujino, D Druzhbin, R Myhill, Y Li, L Wang, T Yamamoto, $\mathrm{N}$ Miyajima, $\mathrm{T}$ Kawazoe, $\mathrm{N}$ Nishiyama, $\mathrm{Y}$ Higo, Y Tange, T Katsura, Generation of pressures over 40 GPa using Kawai-type multianvil press with tungsten carbide anvils, Rev. Sci. Instr. 87, 024501 (2016).

[17] E Ito, D Yamazaki, T Yoshino, H Fukui, T Katsura, Y Tange, K Funakoshi, Pressure generation and investigation of the post-perovskite transformation in $\mathrm{MgGeO} 3$ by squeezing the Kawai-cell equipped with sintered diamond anvils, Eath Planetary Sci. Lett. 293, 84 (2010). 